\title{
CDTRB: QoS based Dynamic Topology Control for Ad- Hoc Network
}

\author{
Jitendra Tiwari \\ Department of Computer Science \& Engineering \\ SDBCT Mhow Road, Indore, (M.P), India \\ Reserach Scholar, (PG)
}

\author{
Jasneet Kour \\ Department of Computer Science \& Engineering \\ SDBCT Mhow Road, Indore, (M.P), India \\ Professor, CSE Dept.
}

\begin{abstract}
MANET is an infrastructure less ad hoc network in which nodes can move freely between specific regions. In this region multiple nodes can communicate with each other. Due to this heavy movement of nodes the topology of the connections is continuously changing within a specific transmission range of the network. To handle this dynamic behaviours or node, efficient topology control mechanism has to be selected according to the environment requirements. These mechanisms can be separated according to their use like mobility sensitive, energy awareness, QoS based etc. An efficient protocol is that which dynamically handles all this issues effectively with better use of bandwidth. This work proposes a new CDTRB based topology control mechanism satisfying the QoS requirements. During the work a survey on various algorithms which is based on above parameters is measured. At the initial level of research the approach seems to provide effective result in near future.
\end{abstract}

\section{Keywords}

MANET, CDTRB (Connectivity, Data Transfer \& Residual Bandwidth), Topology Control (TC), QoS, Mobility, Power Conservation Consistent, Dynamic Topology;

\section{INTRODUCTION}

A group of movable node having wireless type of network between them is known as mobile ad-hoc network (MANET). This type of network is not an infrastructure based network. In this all the mobile nodes can acts as a router for data transmission between multiple nodes within a particular range. Environmental condition of mobile nodes can always affects this transmission and among them mobility is a important factor. Due to which the link can be break due to location updates of the nodes. So for distance location the transmission can be done via third intermediate device which helps in relaying the packet $\&$ routing. Due to all this effective factors \& condition of mobility, the MANET had a wide range of application areas like military, disaster relief, business \& home automation systems.

For an improved MANET architecture various factors can work simultaneously like power utilization, bandwidth utilization, location updates, reliability, security, quality of service (QoS) etc. Among all of them the QoS based topology control (TC) is a primary concern due to frequent changes \& updates in these kind of network scenarios. Quality of service (QoS) refers to resource reservation control mechanisms rather than the achieved service quality. QoS is the ability to provide different priority to different applications, users, or data flows, or to guarantee a certain level of performance to a data flow. The topology of a multi-hop wireless network is a "set of communication links between node pairs used explicitly or implicitly by routing mechanisms". A topology can depend on uncontrollable factors such as node mobility, weather, interference, noise as well as controllable factors such as transmission power, directional antennas and multi-channel communications [1]. The topology control having good QoS will always depends upon various factors \& consideration failure of which leads to wastage of utilization, channel capacity \& communication cost. The design of this topology control should implicitly holds some characteristics for two major scenarios like if the topology is too sparse then the network can get partitioned. However, topology control can provide better control over network resources such as battery power and reduce redundancy in network communications.

In MANET both of the above parameters can be used to understand the mobility principle effectively. Thus the mobility causes related movement of nodes which can break links and thus change the topology and this may result in partitioning of the network. Once the network is partitioned none of routing/broadcast protocol can be successful and very rare chances to form the connected network. For example [2], suppose node $\mathrm{a}$ and node $\mathrm{b}$ are neighbours in the network at time $t$, but somehow move out of the communication range at time $t+\Delta t$. During this period routing is unaware of this broken link and node a still forward packets to $b$ and it will never reaches to node $b$. To keep the topology unchanged without disconnecting any node within the network needs topology management scheme. Effective Topology control will improve the performance and the capacity of Mobile Ad-hoc networks by building reliable network structure [3]. In this paper we are focusing our research concern to both the above mentioned issues of QoS \& topology control implementation of which expects the effective results in future.

\section{BACKGROUND}

The goal of QoS provisioning is to achieve a more deterministic network behaviours, so that information carried by the network can be better delivered and network resources can be better utilized. The QoS parameters differ from application to application e.g., in case of multimedia application bandwidth, delay jitter and delay are the key QoS parameters [4]. After receiving a QoS service request, the main challenges is routing with scalable performance in deploying large scale MANETs. This scalability always needs various topological control algorithms. Most of the work on topology control has dealt with achieving connectivity with node selection as a secondary problem. The primary problem usually attempts to find topologies to minimize power consumption and increased network lifetime. This simultaneous working of QoS \& topology control will always lead their effects on performance of packet delivery fraction $\&$ end to end delays [5] 
The TC methods can be divided into two major areas: homogeneous process of decision control for output. It is based on homogenous range assignment, where all the network nodes have to use the same transmitting range. This case is known as Critical Transmission Range (CTR) problem, which reduces the original TC problem to simpler problem of determining the minimum value of transmitting range such that the network wide property is satisfied. Finding of CTR is the approach reminding centralized solution of TC problem

In non-homogeneous topology control, nodes are allowed to choose different transmitting ranges. Range assignment decision of each node is in this case based on local information about its own neighbourhood. Therefore the decision should be at most almost (locally) optimal, which implies that it is harder to prove the satisfaction of network wide property. Nonhomogeneous TC could be classified into three main categories arising from the type of information that is used to provide TC problem solution. These categories are presented in ordering according to the accuracy of information about node's neighbourhood

\section{Types of Topology Control (TC)}

Topology control can be further divided into three main area:-

Location Based: This approach is based on very accurate information about node positions. Direction Based: When precise position is not available to nodes another group of algorithms could be used which relies on the ability of nodes to estimate the relative direction of their neighbours. This information is less accurate than the previously introduced location based information.

Neighbour Based: This TC protocols rely on node's ability to discover neighbouring nodes within the maximum transmitting range. For this class of methods is crucial the possibility to determine the number and identity of nodes in full power range and to sort these neighbouring nodes in some way. Their algorithm describes that a node increases its transmission power until it finds a neighbour node in every direction based on directional information. But the question remains unanswered if, none of the neighbour node does not exist within the maximum transmission range of the node [6].

The topology control algorithms may be categorized as (a) centralized and (b) distributed in nature. These are some schemes shown for topology control [1]:-

\section{- Centralized Topology Control Algorithms}

Relative Neighbourhood Graph (RNG), Minimum Spanning Tree (MST), Connect, Noble Topology Control Algorithm (NTC), Minimum Radius Graph (minR), Biconn-Augment

\section{- Connectivity Aware Distributed Topology Control} Algorithms

Local Information No Topology (LINT), Local Information Link-State Topology (LILT), Distributed Relative Neighbourhood Graph (Dist-RNG), Distributed Novel Topology Control Algorithm (Dist-NTC)

\section{- Capacity Aware Distributed Topology Control Algorithms}

MobileGrid, Common Power level (COMPOW)

\section{RELATED STUDY}

MANET is a type of dynamic changing nature \& having a varying topology condition at various stages of transmission. There have been many research efforts to provide QoS support in MANETs. Various authors categorizes these efforts into QoS models, QoS resource reservation signalling, QoS routing, and QoS MAC, and provides an overview of how these different components can work together to deliver QoS in MANETs.

In MANET, QoS based TC has no use of existing wired routing protocols. It requires specialized routing schemes which are classified into three categories based on topology update [7]: i) Table driven, ii) On-demand, iii) Hybrid routing protocols. The table driven routing protocol is also known as proactive routing protocol, in this protocol route to every node in the network is maintained in the routing table. Even if route is not required each node maintains the route to other nodes in the network. In case of reactive routing protocol source discovers the route to the destination only if it has some data to send. In hybrid it combines the best features of proactive and reactive routing protocol.

Various approaches is proposed over the last few years which takes this mobile topology change as a prime concern. In the paper [8] mobility-sensitive topology control method is proposed that extends many existing mobility-insensitive protocols. Two mechanisms are introduced: consistent local views that avoid inconsistent information and delay and mobility management that tolerate outdated information.

In the paper [9] the author proposes a novel QoS based AODV protocol for topology control with an improved packet delivery ratio. It extends the AODV Route Request (RREQ) packet to include the following fields: minimum bandwidth, maximum bandwidth and bottleneck bandwidth. The first two fields specify the bandwidth requirements of the application and are populated by the source node. At the initial level of their simulation results it seems to be a effective mechanism.

During this period of research the authors also focused on various interrelated issues of QoS based TC like peer to peer communication [10] in which a new protocol DACME is a probe-based admission control mechanism that performs endto-end QoS measurements according to the QoS requirements of multimedia streams.

In the paper [11] the author proposes a flexible QoS model for MANETs (FQMM) which considers the characteristics of MANETs and combines the high quality QoS of IntServ and service differentiation of DiServ. Salient features of FQMM include: dynamics roles of nodes, hybrid provisioning and adaptive conditioning. Preliminary simulation results show that FQMM achieves better performance in terms of throughput and service differentiation than the best effort model.

In case of multi-beam smart antennas, the network topology needs to be adjusted dynamically by adjusting the beam width and beam directions to minimize interference and to maximize the number of possible concurrent network communications. Thus the author extends this concept to create an Adaptive QoS Topology Control (AQTC) [12] System using Smart Antennas. It uses a cross-layer approach to control the topology dynamically where the topology control layer sits between the MAC and the routing protocol. The performance of protocol has been evaluated using extensive simulations. AQTC always forms a topology to facilitate the current communications and improves the network throughput and end-to-end delay. 
Some of the authors also focused their work on cooperative communication. Most existing works on cooperative communications are focused on link-level physical layer issues. Consequently, the impacts of cooperative communications on network-level upper layer issues, such as topology control, routing and network capacity, are largely ignored. Topology control focuses on network connectivity with the link information provided by MAC and physical layers. There are two aspects in a network topology: network nodes and the connection links among them. This paper proposes a CapacityOptimized Cooperative (COCO) topology control scheme to improve the network capacity in MANETs by jointly considering both upper layer network capacity and physical layer cooperative communications [13].

So many other approaches for QoS is been proposed by various researchers. Some of these are given in [14] like adaptive routing, fair queuing, CADAR, Two level QoS, AOQR, QoS GRID etc. All this approaches made an intermediate decision at various layers of network model like physical, MAC, Network, transport \& application. They all are dealing with dynamic environments having varying topology due to mobility of nodes Some of the authors had also worked on long distance topology control [15], QoS virtual backbone (QoS-VBB) [16], distributed admission control D-AC [17], for more than 15 hops of nodes.

\section{Topology control with mobility}

Motion of the node in MANET comes under the problem domain of mobility. In this the participating nodes can be a mobile node like cell phone, PDA, laptops \& other devices. It may vary from static to dynamic changes \& scenarios. At the initial level the TC is proposed for dealing with static environment \& by applying it periodically we are getting the required results. But for a minute change of time it may be affected unevenly. So as the mobility of the nodes increases the lifetime of TC algorithms can be decreased

By taking the above case the work can say that controlling the topology is a kind of dynamic nature method. It changes according to the motion \& speed of the node. As the speed of the node increases the computation becomes more complex. So it should acquire the property of adapting the fast \& uncontrollable changes

While designing the new dynamic TC, taking only connections as parameters can causes heavy failure because it may be disconnected very fast motion. Therefore algorithm should just try to ensure connectivity for most of nodes and for most of network life time. Several widely used mobility models were introduced in the past, which allow both simulation on individual nodes movement and statistical analysis of behaviour of whole mobility model [1].

\section{- Local Information Link-State Topology (LILT)}

LILT is another algorithm/heuristic which exploits the global topology information for recognising and repairing network partitions. There are two main parts of LILT, Neighbour Reduction Protocol (NRP) and Neighbour Addition Protocol (NAP). NRP reduces the transmission power to maintain the node degree around a certain configured value where as NAP increases the transmission power to establish additional links. When executing NRP and NAP a node receiving a routing update determines whether it is connected or bi-connected. If a node does not receive any updates then it may be in a disconnected state. If the topology is bi-connected then no action is taken. If the topology is disconnected then the node increases its transmission power to the maximum possible value. If the topology is connected and not bi-connected, the node tries to achieve bi-connectivity. Bi-connectivity is reached by evaluating the closest 'articulation point' (AP). An AP is a node whose removal will partition the network.

\section{Energy efficient topology control}

Energy efficiency is an important factor needs to be addressed because changing the scenarios may lead us to lower the energy of devices. It must be preserves by taking the effective utilization of bandwidth. In some cases the QoS parameters can also be applied with this. Two energy efficient QoS topology control algorithms are provided in the paper [8].

\section{- MST based QoS topology control algorithm}

This method uses a minimum spanning tree (MST) for QoS topology controlling. Since we aim to reduce the total transmission power, the minimal power will be chosen to add a new link. Firstly, all node pairs are sorted in ascending order according to the distances between nodes. Then choose the shortest link which does not exist in the current topology and increase transmission power of the node until it can connect with another node along the link. Finally, some calculations leads to determine whether the flows can meet the QoS requirement in the new topology. If not the case, iterate this operation until find a QoS routing or all nodes in the network reach their maximal transmission power $P$.

\section{- MIP based QoS topology control algorithm}

In this method the author consider a minimum incremental power (MIP) tree for QoS topology controlling. Similar to MST, new nodes are added to the tree one at a time until all nodes are included in the MIP tree. However, it is based on the standard Prim algorithm with fundamental difference. Whereas the inputs to Prim's algorithm are the link costs, MIP must dynamically update the costs at each step to reflect the fact that the cost of adding new nodes to a transmitting node's list of neighbourhoods is the incremental cost. Considering node $u$ is already in the tree and node $\mathrm{v}$ is not yet in the tree, the pair $(\mathrm{u}, \mathrm{v})$ which results in the minimum value of the incremental transmission power will be selected.

\section{SURVEY EXTRACTION}

According to the various network conditions network topology can be controlled by the transmitting power \& mobility sensitivity at each node $\&$ the topology can directly affects the QoS factors in the network. So the TC needs to be defined in such a way that it is not too dense (nodes have more neighbours), there would be more choices for routing, but the power consumption of the system would be high with less mobility. On the other hand, if the topology is too loose (with less edges), there would be less choices for routing (hence, some nodes could be overloaded) and the average hop-count between end-nodes would be high. Our goal is to find a balanced topology that can meet end-users QoS requirements and has minimum energy consumption and high mobility.

Thus, this paper identifies the above mentioned issues of topology control with effective network condition to be managed. Some of the futuristic solution has to be addressed to solve the given issues.

Identified Work I: Consistent logical connections can be managed by using synchronous or asynchronous messages. All the nodes must use same location information to select their logical neighbours to assure similar topology condition for 1 effective transmission to their nearest hops. By this partition of 
the network is avoided. For this the location information must be complete \& connection \& power status has to be known accurately.

Identified Work II: If the above given information is not complete then some of the novel mechanism needs to be added on like delay \& handshaking protocol for complete information. Some of the existing location protocols can also be used e.g. LAR to identify the accurate updations regarding the mobility. For heavy mobility condition a buffer zone can be created which deals with high \& low mobility conditions.

Effective simulation, study \& comparison lead the work in correct direction by which QoS can be achieved with topology control.

From the distributed topology control algorithms discussed, LILT incorporates the neighbour adaption approach of LINT, Mobile Grid and Dist-NTC. LILT is also more adaptive to different topology distributions as it tries to maintain connectivity with the APs and keep the network in a biconnected state, thereby improving the network's fault tolerance. The overheads of the algorithms are comparable to other distributed approaches which provide an advantage over other algorithms.

\section{PROBLEM DEFINATION QoS Based TC}

In existing TC protocols, the network is connected with all the nodes under the smaller range of transmission. In this every other node selects its nearest neighbours within its transmission range. This selection of neighbours is based on its nearest hop information of location, energy, connectivity \& number of nodes. This grouping of short range neighbours nodes connected with each other is known as consistent topology. So its needs to be updated synchronously to the changing network conditions. During this changing condition some of the nodes can be moved out of the network $\&$ needs to be updated. The node which is still connected with above consistent group is known as active topology group. Also the parameters related to mobility had some of the QoS factors affecting to that directly. These must be taken into consideration with connectivity \& power.

The above discussion leads to two related issues in topology control:

(i) Taking the actual network condition under the connected transmission ranges, some methods needs to be given which ensures that consistent topology is generated from TC protocol.

(ii) After considering this consistent topology how motion, connectivity, \& power can be maintained for active topology condition.

\section{PROPOSED CDTRB ALGORITHM}

The proposed approach works as an effective topology control mechanism taking QoS parameters as a major concern. Several problems and their corresponding solutions related to QoS topology control and strongly connectivity topology control in wireless ad hoc and sensor networks are discussed. Specifically, topics discussed include: (1) QoS topology construction algorithms in both homogeneous and heterogeneous networks with respect to different optimization objectives; and (2) how to construct strongly connected network topology under both nonrestricted and restricted maximum transmission power.
LINT (Local Information No Topology) and LILT (Local Information Link-state Topology), were proposed for adaptively adjusting node transmitting power to maintain a connected topology in response to topology changes. But, neither LINT nor LILT can guarantee the connectivity of the network

CDTRB (Connectivity, Data Transfer, Residual Bandwidth) Step (i) Initialize with Maximum Connectivity (Max Power),

Data Transfer (Complete Status) and Residual Bandwidth

(Required) // Parameter of work for QoS based distributed topology control

Step (ii) Verify Path (Path value=== Neighbour Node)

// path value contains value or all node and status

Step (iii) Update Routing Table// For Each Neighbours

Step (iv) Method-1

Data Status () // Check Data Transfer Status \& Behaviour

\section{\{}

Check Packet Status (Must Be Complete)

If (Node Receive Packet== Full)

Forward the Packet;

Else

Weight for Completion;

If Packet Received Completely

Then Forward it (Set Forwarding Status to 0 from 1)

// Each Node can forward packet Only Once, Repeat Packet may cause battery Drops. \}

Step (v) Method-2

Power Status () //Check Power and Connectivity Status for Each Node

Remaining Power=Sum of Power of all Node- Power Used for Forwarding

For Further Transmission Checks

Remaining Power is Greater Than Decided Threshold, Continues Communication

Else Search for New path// Distributed Topology Updated

Update new Node and Link (Status==Completely Connected) \}

Step (vi) Method-3

//Read Link Quality Bit for All Intermediate Node

Link Quality Value ()

\{

Residual Bandwidth $=($ Total Bandwidth Available - Current Bandwidth Utilized)/ Total Bandwidth for Channel

For Each Path to its Neighbours

Monitor (Residual Bandwidth) 
If (Flow $==$ Accepted $\&$ Identified bandwidth $>$ Required Bandwidth)

Set Link Quality=1

Else $/ /$ Flow $==$ Accepted $\&$ Identified Bandwidth $<$ Required Bandwidth

And If (Flow==Rejected)

Set Link Quality <0

\} // Link Quality Values Varies From $\{-1,1\}$.

Step (vii) Repeat the above Steps and collect the data

Step (viii) Calculate Average Quality Path $=$ (Route Existence (Connectivity and Power) + Data Status (Complete) + Residual Bandwidth $) / 3$

Step (ix) Method-4

Select the Best Suited Path for Transmission \{

Average Quality Path>= Min Threshold

Do the Above for Period P \}

Step (x) For New Element in Average Quality path

Step (xi) Step (xi) Update (Delete Old Path, Add New Path)

Step (xii) Set Status to Bi-Directional

// For Complete Coverage \& Topology Complexity Reduction

Step (xiii) Exit;

\section{EXPERIMENTAL EVALUATION}

\section{QoS Based Performance Evaluation Metrics}

To calculate the better topology control we need to add some of the quality measures which analyse various factors for improved bandwidth utilization, power saving \& strong connections. For these performance evaluations, metrics includes the following QoS parameters such as PDR (Packet Delivery Ratio), Throughput, End to End Delay, Routing overhead and Jitter.

1) Packet Delivery Ratio (PDR): also known as the ratio of the data packets delivered to the destinations to those generated by the CBR sources. This metric characterizes both the completeness and correctness of the routing protocol also reliability of routing protocol.

2) Average End to End Delay: Average End to End delay is the average time taken by a data packet to reach from source node to destination node. It is ratio of total delay to the number of packets received.

3) Throughput: Throughput is the ratio of total number of delivered or received data packets to the total duration of simulation time.

4) Normalized Protocol Overhead/ Routing Load: Routing Load is the ratio of total number of the routing packets to the total number of received data packets at destination.
5) Jitter: Jitter describes standard deviation of packet delay between all nodes.

\section{CONCLUSION}

Various approaches had been proposed for topology control according to the requirements during the last few years. Among all of these QoS, Mobility \& energy conservation based approaches is getting popularity day by day. To select the best one between them which satisfy all the user requirements is a typical task. Thus this paper gives a wide selection criterion after which it becomes easy to select better approach. Topology control in ad hoc network will dynamically improves the performance of the network by specific orientation of nodes towards these parameters of quality. Among the various mechanism we have mentioned approaches for dynamic topology control with a hop-by-hop routing protocol which increases the network throughput and reduces the end-to-end delay. Thus to analyse the effective TC algorithms above mentioned metrics of QoS needs to be calculated. In this way the required TC can be find out.

\section{FUTURE WORK}

Some problems and concepts that remain unaddressed can be performed in future work. It will focus on other approaches and different aspects of dynamic topology control and evaluating their performance under different network conditions. Comparison of the various simulation results leads to better analysing the results.

\section{REFERENCE}

[1] Gaurav Srivastava, Paul Boustead, Joe F.Chicharo, "A Comparison of Topology Control Algorithms for Ad-hoc Networks", in School of Electrical, Computer and Telecommunication Engineering, University of Wollongong, NSW, Australia.

[2] S. S. Dalu, M. K. Naskar \& C. K. Sarkar, "Implementation of a Topology Control Algorithm for Mobile Ad hoc Networks Using Pursue Mobility Model", in Journal of Applied Computer Science, ISSN:1843-1046, Vol (2), no.2, 2008,

[3] S. Karunakaran and P. Thangaraj, "Topology Control Using Efficient Power Management", in Journal of Computer Science, Science Publications, ISSN 1549-3636, Vol 7, No 4:pp 561-567, 2011.

[4] Rajneesh Kumar Gujral \& Manpreet Singh, "Analyzing the Impact of Scalability on QoS-aware Routing for MANETs", in IJCSI Issues, ISSN (Online): 1694-0814, Vol. 8, Issue 3, No. 1, May 2011

[5] Koushik Majumder \& Subir Kumar Sarkar, "QOS Parameter Based Performance Study of the Gateway Discovery Approaches for MANET" in International Journal of Computer Applications (IJCA), 2011.

[6] Surendra S. Dalu, M.K Naskar \& C.K Sarakar, " Hardware Implemenatation of a Topology Control Algorithm for MANET's Using Nomadic Community Mobility Model", in Journal of Theoretical and Applied Information Technology, 2009.

[7] S. R. Biradar, Subir Kumar Sarkar \& Puttamadappa, "Analysis QoS Parameters for MANETs Routing Protocols", in (IJCSE) International Journal on Computer Science and Engineering, Vol. 02, No. 03, 2010. 
[8] Jie Wu \& Fei Dai, "Mobility-Sensitive Topology Control in Mobile Ad Hoc Networks" in International Parallel and Distributed Processing Symposium,IPDPS- 2004

[9] Vishal Garg1 \& Chandan Kapoor, "A Survey of QoS parameters through reactive routing in MANETs" in IJCEM International Journal of Computational Engineering \& Management, ISSN (Online): 2230-7893, Vol. 13, July 2011

[10] Carlos T. Calafate, Juan-Carlos Cano, Pietro Manzoni \& Manuel P. Malumbres, "A QoS architecture for MANETs supporting real-time peer-to-peer multimedia applications", in Polytechnic University of Valencia (UPV), Valencia, Spain.

[11] Hannan Xia, Winston K.G. Seah, Anthony Lo \& Kee Chaing Chuaa, "Flexible Quality of Service Model for Mobile Ad-Hoc Networks", in National University of Singapore.

[12] Sk. Mohammad Rokonuzzaman, Ronald Pose and Iqbal Gondal, "A Cross-layer Approach for QoS Topology Control in Wireless Ad Hoc Networks" in IEEE , 978-14244-4547-9/09, 2009.
[13] Quansheng Guan, Shengming Jiang, F. Richard \& Yu "Topology Control in Mobile Ad Hoc Networks with Cooperative Communications" Univ. of British Columbia, Vancouver, BC, Canada

[14] Winston K. G. Seah, Hwee-Xian Tan, "Quality of Service in Mobile Ad Hoc Networks", in IGI Global National University of Singapore, 2008.

[15] Kitikun Poolsub, Benjaporn Lapwilai \& Dobri Atanassov Batovski, "Topology Control for Long Distance Transmission in Multihop Ad Hoc Networks", in AU J.T. 13(4): 213-222 ,Apr. 2010.

[16] Moussa Ayyash, Donald Ucci \& Khaled Alzoubi, “A Proactively Maintained Quality of Service Infrastructure for Wireless Mobile Ad Hoc Networks" in International Journal of Communication Networks and Information Security (IJCNIS), Vol. 2, No. 2, August 2010.

[17] Pushpavalli M \& Annitha N, "A Distributed Admission Control Based Quality of Service in MANET's", in International Journal of Engineering Trends and Technology,ISSN: 2231-5381 Volume 4, Issue 4- 2013 